\title{
COMAROSTAPHYLIS ARBUTOIDES (ERICACEAE) EN EL CENTRO Y OCCIDENTE DE MÉXICO
}

\author{
Martha GonzÁlez-Elizondo ${ }^{1,}$ M. Socorro GonZÁlez-Elizondo ${ }^{1}$ \\ y Sergio Zamudio ${ }^{2,3}$
}

${ }^{1}$ Instituto Politécnico Nacional, Centro Interdisciplinario de Investigación para el Desarrollo Integral Regional, Unidad Durango, Sigma 119, Fracc. 20 de Noviembre II, 34220 Durango, Durango, México.

${ }^{2}$ Instituto de Ecología, A.C., Centro Regional del Bajío, Apdo. postal 386, 61600 Pátzcuaro, Michoacán, México.

${ }^{3}$ Autor para la correspondencia: sergio.zamudio@inecol.mx

\section{RESUMEN}

Comarostaphylis arbutoides, previamente conocida en México de los estados de Guerrero, Oaxaca y Chiapas, así como de Centroamérica, se registra adicionalmente para Jalisco, Querétaro e Hidalgo, lo que extiende en casi $500 \mathrm{~km}$ hacia el norte su distribución conocida. Se amplía la descripción de la especie incluyendo la variación encontrada en las nuevas poblaciones y se clarifican sus diferencias de Comarostaphylis lanata y C. sharpii.

Palabras clave: Comarostaphylis, flora, región del Bajío.

\begin{abstract}
Comarostaphylis arbutoides, previously known from Guerrero, Oaxaca, and Chiapas in Mexico, as well as from Central America, is additionally recorded from Jalisco, Querétaro, and Hidalgo, extending its known distribution area by almost $500 \mathrm{~km}$ northwards. The description of the species is broadened to include the morphological variation found in the newly registered populations, and differences from Comarostaphylis lanata and C. sharpii are clarified.
\end{abstract}

Key words: Bajio region, Comarostaphylis, flora. 
El género Comarostaphylis Zucc. (Ericaceae, Arbutoideae), con 12 especies y un total de 19 taxa, se distribuye en regiones con climas templados y fríos desde el sur de California hasta el oeste de Panamá (Diggs, 1995). Su mayor diversificación se presenta en las montañas de México. Durante la revisión de la familia Ericaceae para la Flora del Bajío (González-Elizondo y González-Elizondo, 2014) se registraron plantas morfológicamente similares a Comarostaphylis arbutoides Lindl., una especie considerada como endémica de Mesoamérica por varios autores (Breedlove, 1986; Diggs, 1981, 1995; Stevens et al., 2001; Luteyn et al., 2009), pero que se ha registrado también para la Sierra de Taxco, Guerrero (Martínez-Gordillo et al., 2004) y para la Sierra de Juárez, Oaxaca (Zacarías-Eslava y del Castillo, 2010). Las localidades citadas en el presente trabajo extienden la distribución conocida del taxon en casi $500 \mathrm{~km}$ hacia el norte.

Comarostaphylis arbutoides representa a un complejo en el que Diggs (1995) reconoce dos subespecies: $C$. arbutoides subsp. arbutoides, con ramillas, peciolos y envés de las hojas ferrugíneo-tomentosos o tomentulosos, inflorescencias tomentosas a glandular hirsutas y lóbulos del cáliz pubescentes, distribuida desde Chiapas hasta el oeste de Panamá, y C. arbutoides subsp. costaricensis (Small) Diggs, endémica de Costa Rica, con ramillas, peciolos y envés de las hojas glabros, a veces glaucos, inflorescencias glabras o con ligera pubescencia, y lóbulos del cáliz glabros. Comarostaphylis chiriquensis Camp y C. sleumeri Suess. son incluidas por el mismo autor en la sinonimia de C. arbutoides subsp. arbutoides.

Comarostaphylis arbutoides ha sido enlistada en la categoría de "vulnerable" en versiones previas de la IUCN pero no se incluye en la actual (IUCN, 2013). Se cita entre las especies vulnerables tanto para bosques mesófilos (González-Espinoza et al., 2011) como para la Reserva de la Biosfera El Triunfo (Martínez-Camilo et al., 2012). Es también de interés por formar micorrizas arbutoides (Diggs, 1995; Osmundson et al., 2007) asociadas a encinos.

Las plantas de Mesoamérica se conocen de Chiapas, Guatemala, Honduras, Nicaragua, Costa Rica y Panamá, donde crecen en altitudes entre 1200 y 3800 m, en bosques de Quercus o Pinus, bosques de neblina y páramos de altura (Diggs, 1995; Luteyn et al., 2009; Rodríguez et al., 2011). En los estados de Querétaro e Hidalgo se desarrolla entre 1900 y 3050 m, principalmente en encinar arbustivo o chaparral denso, pero también en bosques de encino, pino-encino y pino. En el encinar arbustivo crece con Quercus depressipes, $Q$. greggii, Q. repanda, Abelia grandifolia, Cercocarpus macrophyllus, Nolina hibernica, Berberis hartwegii, Croton ehrenbergii, Garrya glaberrima, Litsea glaucescens y Amelanchier denticulata. Se desarrolla también en bosques de Pinus patula y en bosques de Pinus greggii, en el segundo 
caso combinado con elementos del bosque mesófilo de montaña, como Juglans, Clethra, Cupressus lusitanica, Ilex discolor, Morella cerifera y Brahea moorei. En los bosques de encinos destacan Quercus polymorpha, Quercus affinis, Q. crassifolia y Arbutus xalapensis.

La población de Comarostaphylis arbutoides de Jalisco ha sido citada como Comarostaphylis lanata Small por González Villarreal (1990) y Diggs (1995). Adicionalmente, plantas de Durango que comparten el carácter de hojas coriáceas, lanoso-ferrugíneas en el envés fueron citadas como $C$. aff. lanata Small por González-Elizondo et al. (1991) y como C. cf. sharpii Dorr \& Diggs por Diggs (1995); este último autor hace notar que se requiere más material para una identificación definitiva. Es pertinente aclarar que los individuos de Durango presentan flores de color rosa-intenso a fucsia y hojas más angostas, por lo que se consideran como una especie distinta, actualmente en proceso de descripción.

Las plantas de Jalisco, Querétaro e Hidalgo se reconocen como C. arbutoides por sus características morfológicas, aunque cabe la posibilidad de que estudios moleculares o químicos posteriores pudieran revelar divergencias evolutivas, particularmente en aquellas que presentan la mayor separación geográfica (Fig. 1). Las de Querétaro e Hidalgo presentan ramillas, hojas e inflorescencias con aspecto general similar a $C$. arbutoides subsp. arbutoides, pero son arbustos o arbolitos más bajos (de 0.6 a 2(3) $\mathrm{m}$ de altura, Fig. 3 b), mientras que en Mesoamérica se encuentran como arbustos o árboles de 1 a 20 m (Luteyn et al., 2009); tienen hojas lanceoladas, obovado-lanceoladas y aún obovado-espatuladas, con envés más lanoso (Fig. 2 a, b) y flores amarillo-verdosas (Fig. 3), sin el tinte rosa que es común en ejemplares de $C$. arbutoides de Mesoamérica. Sin embargo, debido a que las discontinuidades morfológicas entre las poblaciones del centro de México en relación con las de Mesoamérica no son discretas, y dado el estado actual del conocimiento del conjunto involucrado, todas se consideran como parte del complejo de $C$. arbutoides.

Las divergencias morfológicas arriba mencionadas se interpretan como resultado del limitado intercambio genético entre las poblaciones. Entre las más notables destacan las de individuos con hojas obovado-espatuladas (Fernández 2519, Zamudio 10348), con aspecto general similar al de Comarostaphylis sharpii, una especie conocida de Tamaulipas. A pesar de la similitud superficial con C. sharpii, las plantas con hojas obovado-espatuladas parecen representar un extremo de variación de $C$. arbutoides s.l., ya que es frecuente encontrar en un mismo individuo hojas lanceoladas y hojas obovado-espatuladas. Un espécimen (Zamudio 15581) tiene hojas serruladas, relativamente cortas y anchas, por lo que podría ser identificado 


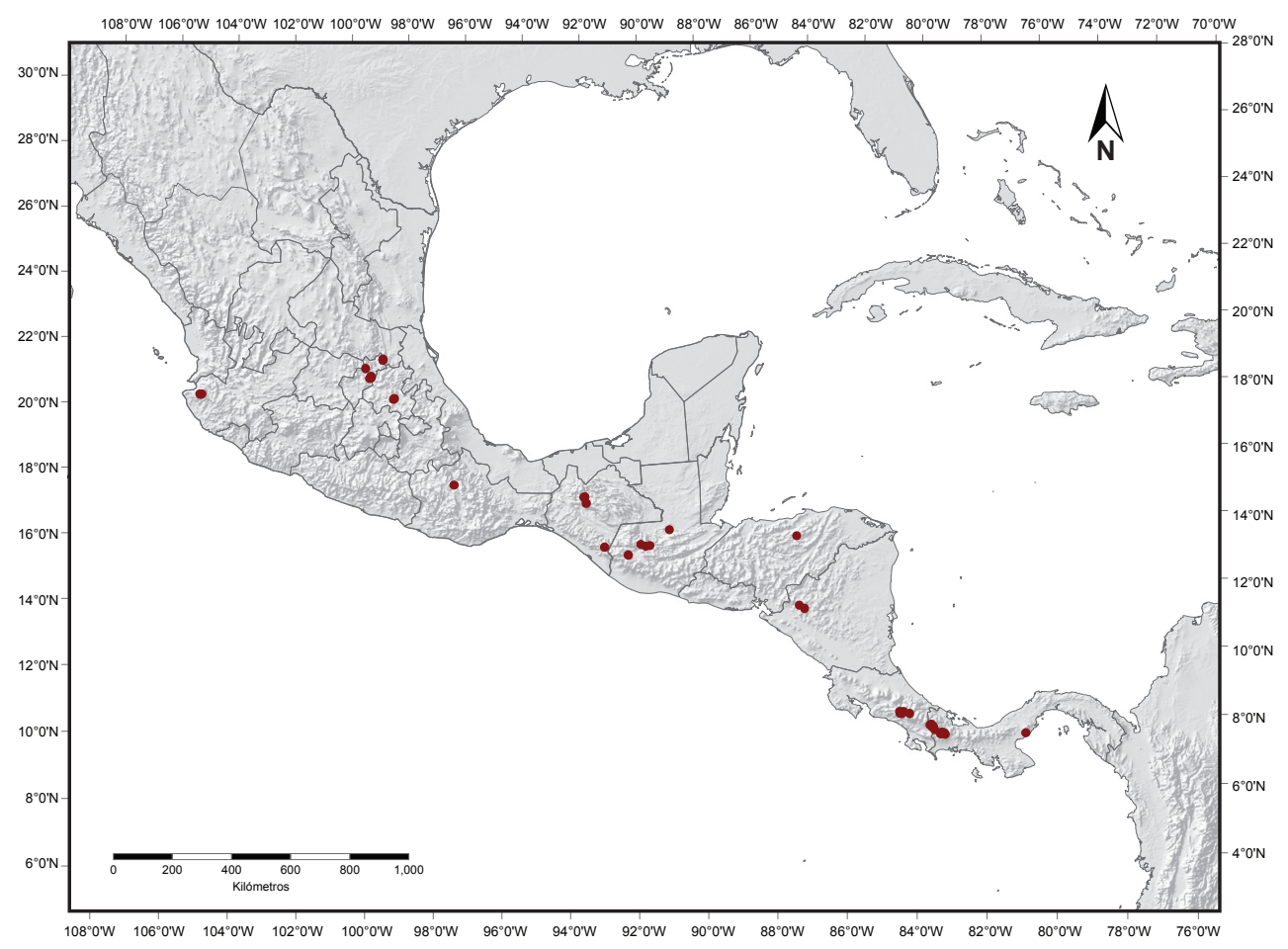

Fig. 1. Distribución de Comarostaphylis arbutoides s.l.

como C. spinulosa (M. Martens \& Galeotti) Diggs subsp. glandulifera Diggs, taxon conocido de Puebla y Oaxaca, del que se distingue por tener hojas más gruesas con serrulaciones irregulares y espaciadas, 3-4 cm (vs. regulares y de 6-9 cm) e inflorescencias más grandes y más densamente hirsuto-glandulares. Las características diagnósticas de C. arbutoides y de las especies con las que ha sido confundida se presentan en el Cuadro 1.

Comarostaphylis lanata (Fig. 4) y C. sharpii (Fig. 5) son endémicas de la Sierra Madre Oriental, la primera de Buenavista, San Luis Potosí y la segunda de Bustamante y de Gómez Farías, Tamaulipas. Otra colecta de Bustamante, Tamaulipas (M. Martínez 00417) parece representar una variante no lanosa de C. lanata por tener las hojas ascendentes, densamente imbricadas, estrechamente elípticas y marcadamente revolutas, flores rojas e inflorescencia hirsuto glandular con tricomas rojos, pero difiere de ésta en carecer de tomento blanco lanoso en el envés de las hojas, el cáliz no densamente tomentoso y la corola glabra. 
González-Elizondo et al.: Comarostaphylis arbutoides de México

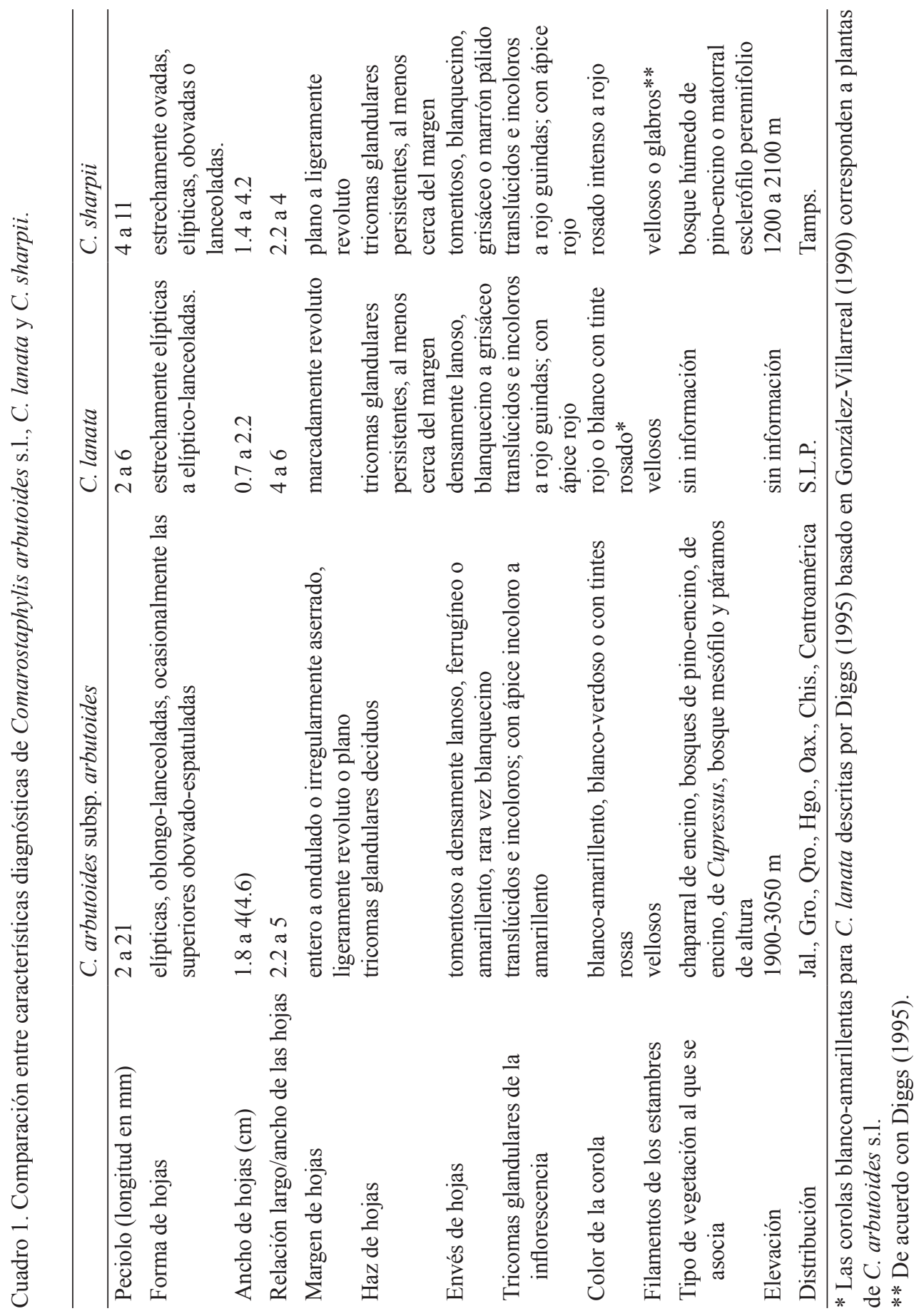


Acta Botanica Mexicana 111: 47-59 (2015)

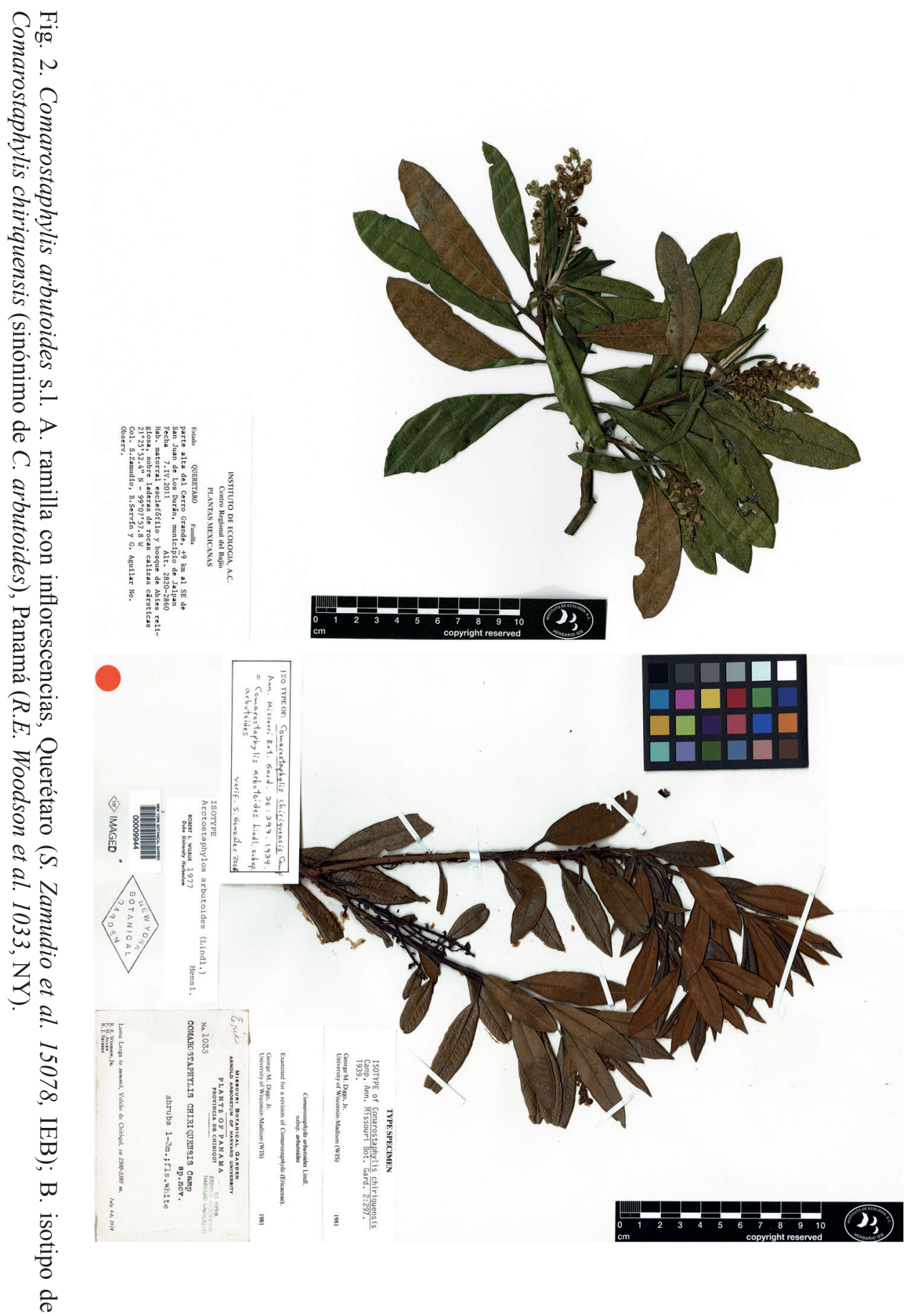



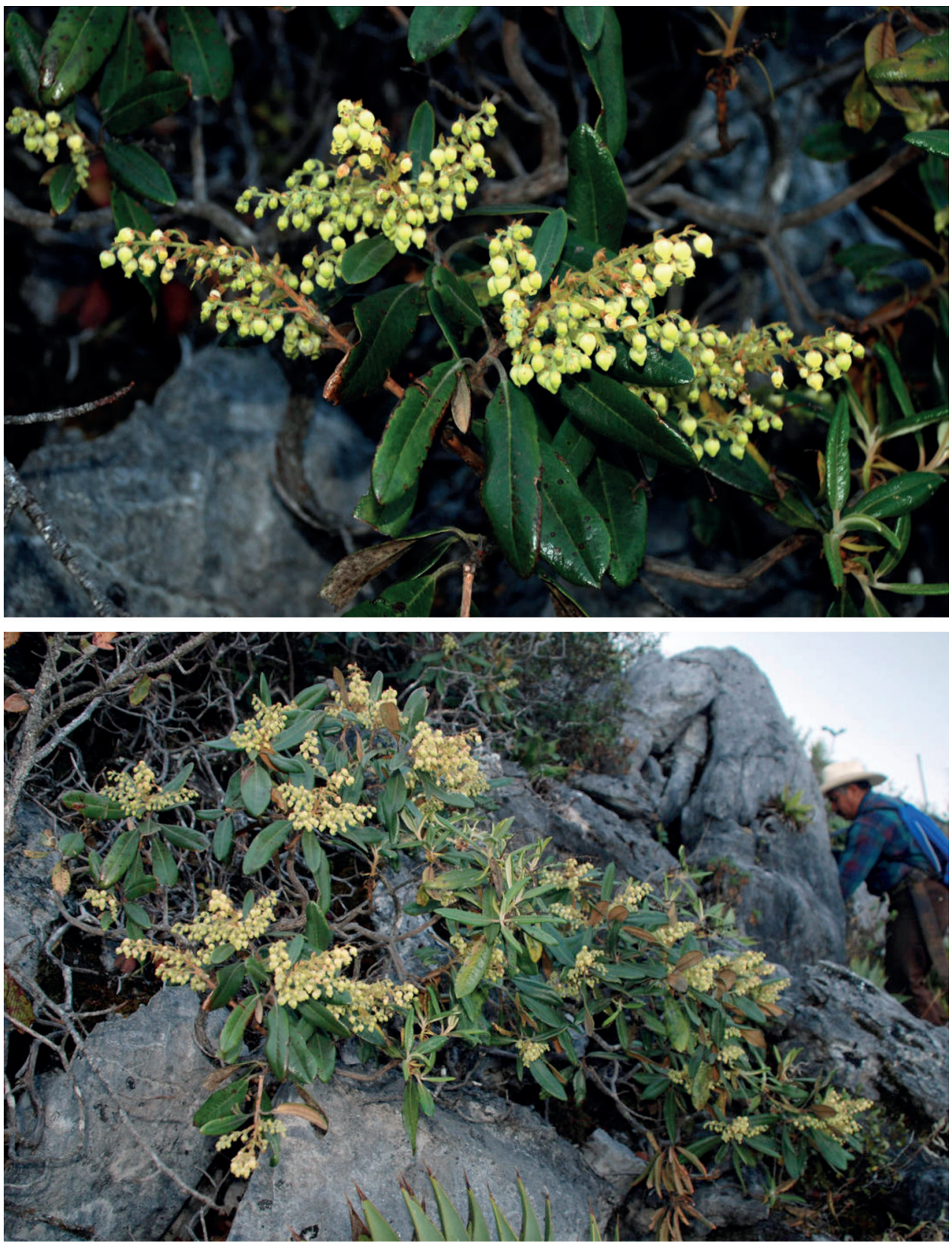

Fig. 3. Comarostaphylis arbutoides s.1. en Querétaro: A. inflorescencias; B. hábito. Fotografías: S. Zamudio. 


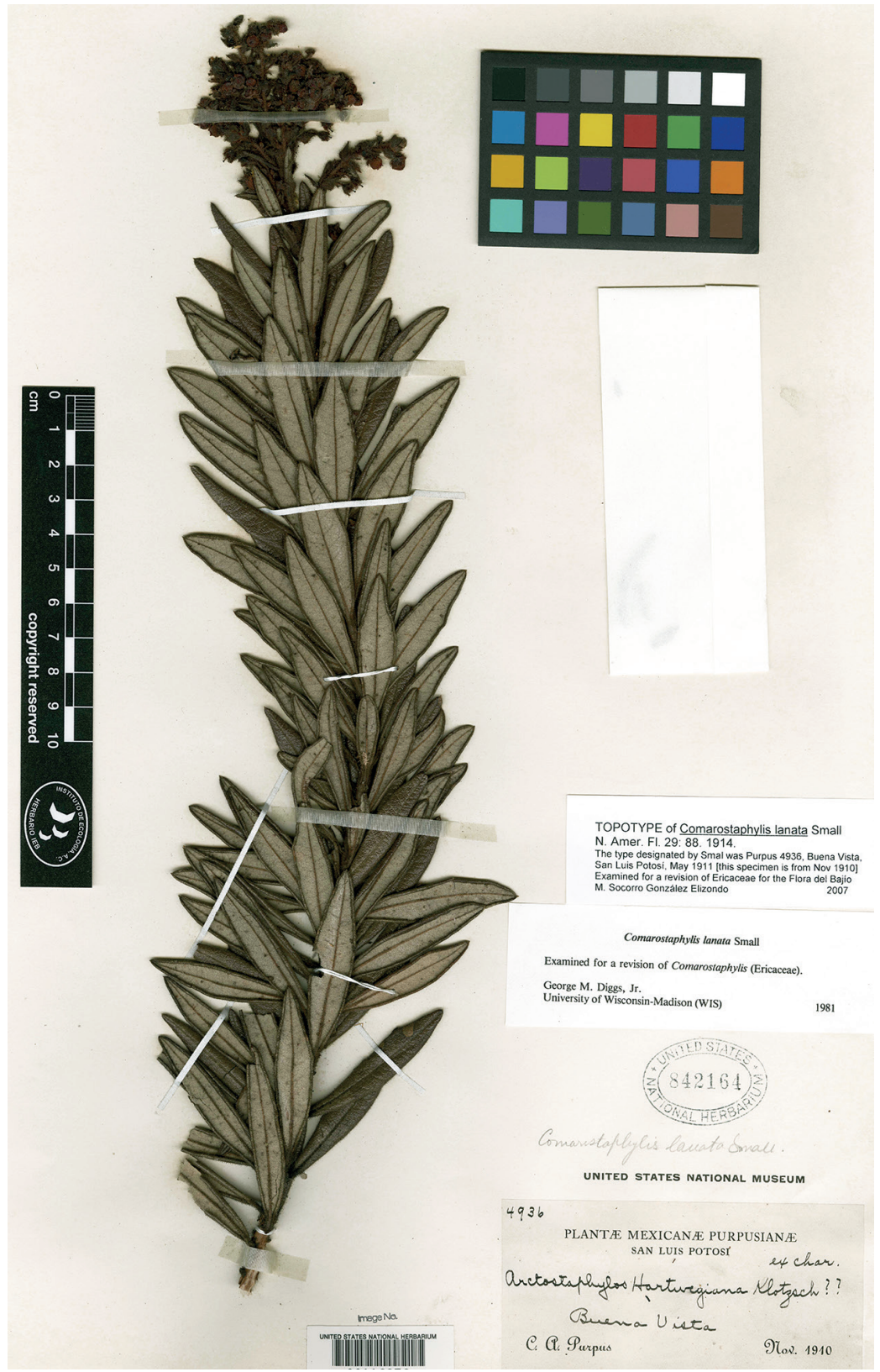

Fig. 4. Comarostaphylis lanata. Imagen de isotipo en US. Aunque el número de colecta corresponde al del tipo, la muestra fue colectada en Noviembre de 1910 y no en Mayo de 1911 (fecha de colecta del tipo). Dado que Purpus colectó en la misma región en 1910 y en 1911, es probable que este espécimen deba considerarse como topotipo. 
González-Elizondo et al.: Comarostaphylis arbutoides de México

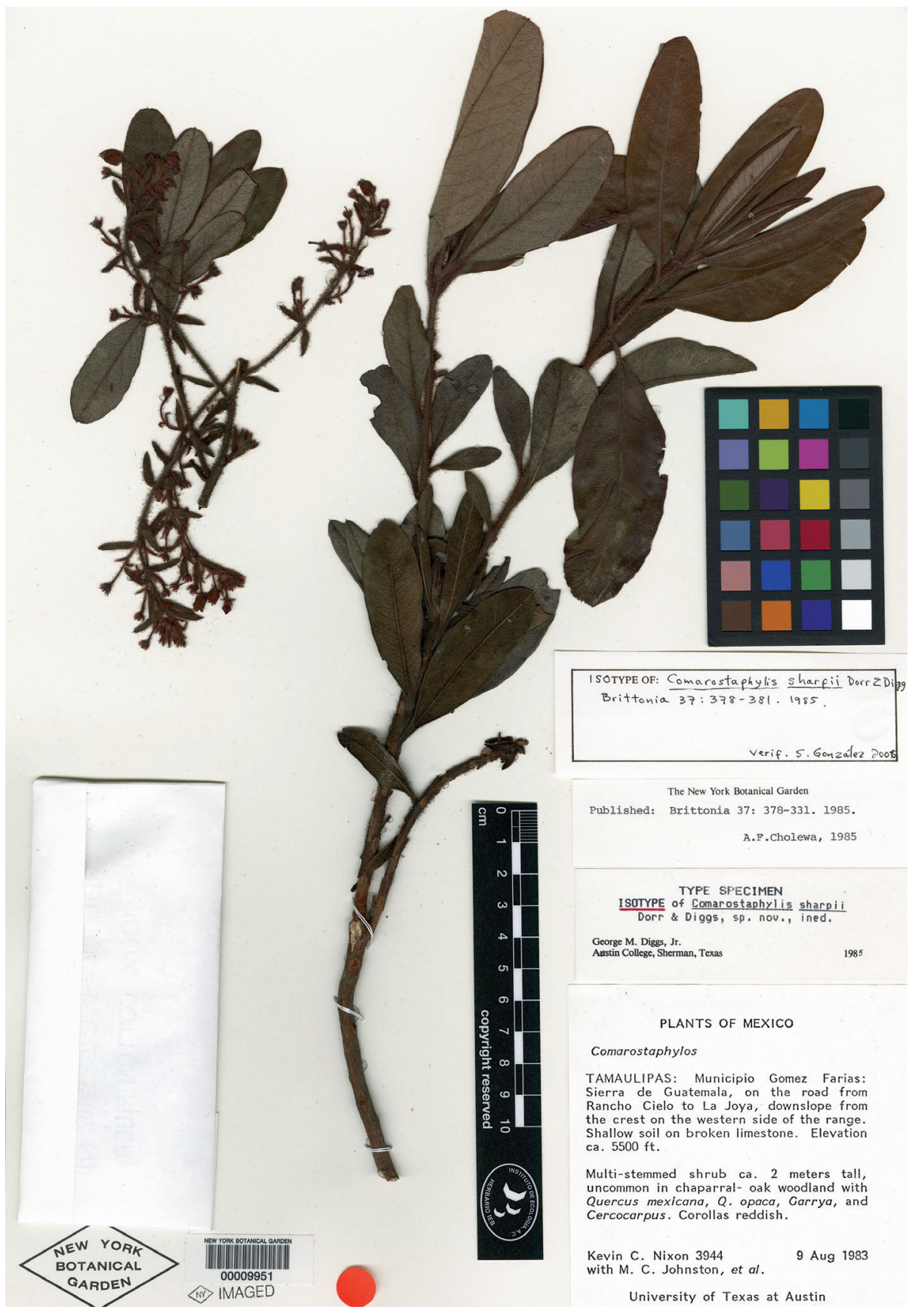

Fig. 5. Comarostaphylis sharpii. Imagen de isotipo en NY. 
A continuación se proporciona una descripción de Comarostaphylis arbutoides subsp. arbutoides incluyendo las características de las plantas del centro y occidente de México (adaptada de González-Elizondo y González-Elizondo, 2014). La amplia variación morfológica y el vasto intervalo altitudinal de las localidades en las que se desarrollan los individuos reconocidos como $C$. arbutoides sugieren que el grupo representa un complejo que requiere de un estudio taxonómico más extenso y profundo para esclarecer su situación taxonómica.

Comarostaphylis arbutoides Lindl., Edwards's Bot. Reg. 29: t. 30. 1843. subsp. arbutoides. Arctostaphylos arbutoides (Lindl.) Hemsl., Biol. cent.-amer., Bot. 2: 278. (lectotipo: GUATEMALA. De material cultivado de semillas colectadas cerca de Quezaltenango (Hartweg s.n. [871] (CGE), designado por Diggs (1995: 157).

Arbusto o arbolito, 0.6 a 2(3) $\mathrm{m}$ de alto (o árbol de hasta $20 \mathrm{~m}$ en Mesoamérica), erecto a patente, muy ramificado, perennifolio; corteza exfoliante en laminillas irregulares, o semipersistente, de color gris o café, la corteza interior de color anaranjado pálido, ramillas jóvenes densamente hirsuto-glandulares, a veces también con tricomas simples rizados, corteza delgada, desprendiéndose en tiras largas; peciolos de 2 a $21 \mathrm{~mm}$ de largo, hirsuto-glandulares o eglandulares o casi eglandulares en algunas plantas de Centroamérica; láminas elípticas, oblongo-lanceoladas a obovadas, a veces las superiores obovado-espatuladas, de (4.5)5.5 a $12.6 \mathrm{~cm}$ de largo y 1.8 a $4.6 \mathrm{~cm}$ de ancho (o angostas, desde $1 \mathrm{~cm}$ de ancho en Mesoamérica), ápice obtuso o agudo a abruptamente apiculado, con frecuencia mucronado, base cuneada, margen entero a ondulado o irregularmente aserrado sobre todo hacia la mitad distal, ligeramente revoluto o plano, coriáceas, bicoloras, haz de color verde olivo o verde obscuro, nítido a opaco, con las nervaduras marcadamente impresas formando una retícula, pubescente, con tricomas glandulares deciduos, envés tomentoso a densamente lanoso con tricomas simples y algunos tricomas glandulares, particularmente a lo largo de la nervadura central, la pubescencia de color amarillento a intensamente ferrugíneo, rara vez blanquecino; nervadura central prominente en el envés; inflorescencias terminales, paniculadas, frecuentemente muy ramificadas, de (4.5)10 a $26 \mathrm{~cm}$ de largo y (3.5)7 a $22 \mathrm{~cm}$ de ancho, el raquis, las ramas y los pedicelos conspicuamente hirsuto-glandulares, con tricomas de hasta $3 \mathrm{~mm}$ de largo, translúcidos e incoloros, con el ápice incoloro a amarillento, bráctea floral deltoide a lanceolada, glabra a densamente pubérula adaxialmente, con tricomas glandulares, aguda a acuminada, glandular-ciliada, pedicelos de (1)3 a 10(20) $\mathrm{mm}$ de largo, bractéolas basales a medias, lineares o linear-lanceoladas, 1 a $7.6 \mathrm{~mm}$ de largo, 
glandular-ciliadas o no; lóbulos del cáliz deltoides a ovado-lanceolados, 1.7 a $5 \mathrm{~mm}$ de largo y de 1.2 a $1.8(3.1) \mathrm{mm}$ de ancho, glabros a pubérulos en el lado adaxial, a veces con márgenes glandular-ciliados; corola urceolada, 5 a $7.5 \mathrm{~mm}$ de largo, $3.5 \mathrm{a}$ $5 \mathrm{~mm}$ de ancho, de color blanco-amarillento, blanco-verdoso o con tintes rosas, finamente papilosa, pubescente a densamente pilosa con tricomas simples, los lóbulos con márgenes auriculados imbricados; estambres con filamentos vellosos, anteras dehiscentes por poros alargados o ranuras de más de $1 / 3$ su longitud, con apéndices de 0.4 a $0.8 \mathrm{~mm}$ de largo o inconspicuos; ovario piloso; fruto de 0.4 a $0.6 \mathrm{~cm}$ de largo y de 0.4 a $0.8 \mathrm{~cm}$ de diámetro, de color rojo cuando inmaduro, púrpura obscuro a negro al madurar, semillas de color ámbar a café-rojizo, superficie reticulada.

Nombres comunes: arrayán, chilú, madroño prieto, nariz de chucho, nariz de perro.

Distribución y hábitat: Se distribuye en Jalisco, Guerrero, Querétaro, Hidalgo, Oaxaca, Chiapas y Centroamérica. Las plantas de Jalisco, Querétaro e Hidalgo habitan en bosques de pino-encino, de encino, de Cupressus, mesófilo de montaña y en chaparral de encino, principalmente sobre rocas calizas aunque en Hidalgo y Jalisco crecen también sobre rocas ígneas. Se desarrollan en un amplio intervalo altitudinal que va de 1900 a $3050 \mathrm{~m}$.

Materiales examinados para el centro y occidente de México: Jalisco. Municipio de Talpa de Allende: Minas de Zimapán, Sierra de Cuale, L. M. González Villarreal y J. A. Pérez de la Rosa 3033 (IBUG), 3036 (CIIDIR, IBUG), 3037 (CIIDIR, IBUG); ibid., R. Ramírez D., J. A. Pérez de la Rosa, Villa V. y R. González Tamayo 816 (IEB); El Caracol, 1-2 km al W de la mina Zimapán, J. A. Pérez de la Rosa 1365 (IBUG); ibid., L. M. González Villarreal 3090 (IBUG, IEB), 3091 (IBUG, IEB), 3465 (IBUG); cerro del Cuale, Cerro Caracol, cerca de la mina del Cuale, $M$. Cházaro et al. 4756 (IEB). Querétaro. Municipio de Jalpan: $7 \mathrm{~km}$ al SW de Jalpan, R. Fernández 2519 (ENCB, IEB, MEXU). Municipio de Landa: 7-8 km al S de San Juan de Los Durán, llano de Las Avispas, B. Servín 946 (CIIDIR, IEB); ladera N del

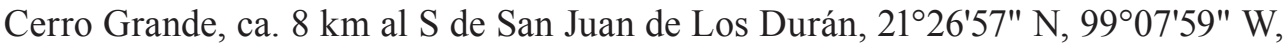
S. Zamudio et al. 14847 (CIIDIR, IEB); ladera N del Cerro Grande, ca. $9 \mathrm{~km}$ al S de San Juan de Los Durán, 2125'59" N, 9908'08" W, S. Zamudio y B. Servín 15011 (IEB); Cerro Grande, 5-6 km al S de San Juan de los Durán, B. Servín 3108 (CIIDIR, IEB); parte alta del Cerro Grande, al SE de San Juan de Los Durán, 2125'52" N, 9907'58" W, S. Zamudio et al. 15078 (CIIDIR, IEB); cima del Cerro Grande, ladera 
W, 2125'57" N, 9908'05" W, B. Servín 3158 (IEB); las abras del Cerro Grande, al NE de la Lagunita de San Diego, E. Carranza 2867 (IEB); Llano Chiquito, $S$. Zamudio et al. 11423 (CIIDIR, IEB); vertientes SE del cerro Piedra de La Cruz, E. Carranza 3174 (CIIDIR, IEB); Municipio de Pinal de Amoles: cañada El Copal, al $\mathrm{N}$ del cerro Pingüical, E. Carranza 1740 (IEB); vertiente N del cerro El Pingüical, E. Pérez y E. Carranza 3550 (CIIDIR, IEB); cerca del Puerto de Canoa, vertiente $\mathrm{N}$ del Cerro Pingüical, J. Rzedowski 48191 (IEB); cima del cerro La Taza, cerca del

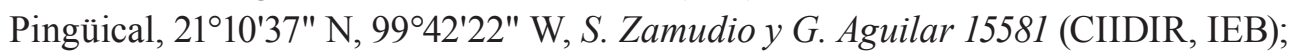
caseta contra incendios forestales y el rancho La Trinidad, S. Zamudio y E. Zamudio 10348 (IEB); ca. de $0.5 \mathrm{~km}$ de Cuatro Palos camino a La Cañada, E. Carranza 2106 (IEB); cerro La Calentura, $\pm 2 \mathrm{~km}$ al $\mathrm{N}$ de El Madroño, E. Carranza 2081 (IEB). Municipio de San Joaquín: 4 km al NE de San Joaquín por el camino a Bucareli, $S$. Zamudio y E. Pérez 7967 (CIIDIR, IEB); alrededores de San Joaquín, R. Hernández et al. 10893 (ENCB, IEB, QMEX); Maravillas-Santo Tomás, cerca de San Joaquín, R. Hernández et al. 11042 (IEB, QMEX); zona arqueológica Toluquilla, S. Zamudio et al. 11395, 11398 (CIIDIR, IEB); ibid., S. Zamudio y S. González 11749 (CIIDIR, IEB). Municipio de Cadereyta: $1 \mathrm{~km}$ al N de El Doctor, sobre el camino a Vizarrón, J. Rzedowski 43076 (IEB); ca. $2 \mathrm{~km}$ de El Doctor por el camino a Altamira, E. Pérez y C. Medina 4996 (IEB). Hidalgo. Municipio de Actopan: Los Órganos, ca. 12 km al SE de San Andrés, S. Zamudio y G. Ocampo 11035 (CIIDIR, IEB); Pico del Fraile, brecha de San José Tepense a San Gerónimo, M. Cházaro et al. 6497 (IEB).

\section{AGRADECIMIENTOS}

Agradecemos al Dr. Jerzy Rzedowski su paciencia y permanente apoyo para concluir este trabajo. Al Dr. George M. Diggs por comentarios sobre la taxonomía de Comarostaphylis. Al Dr. Emmanuel Pérez Calix por apoyo en campo y al Dr. Jorge A. Tena por ayuda en diferentes fases del trabajo. A la M. en C. Martha Beatriz Campos por la elaboración del mapa y a los encargados de los herbarios CAS, CHAP, CHAPA, CIIDIR, ENCB, GH, IBUG, IEB, MEXU, MICH, NY, QMEX, SLPM, UAT y US por el préstamo de materiales y/o facilidades para consulta. A la Comisión de Operación y Fomento de Actividades Académicas por los estímulos a la investigación. El trabajo fue desarrollado con apoyo económico del Instituto Politécnico Nacional (proyectos 20130850 y 20141193) y del Instituto de Ecología, A.C. (Cuenta 20006), del Consejo Nacional de Ciencia y Tecnología y de la Comisión Nacional para el Conocimiento y Uso de la Biodiversidad. 


\section{LITERATURA CITADA}

Breedlove, D. E. 1986. Flora de Chiapas. Listados Florísticos de México 4: 1-246.

Diggs, G. M. 1981. Systematic studies in the Arbuteae (Ericaceae, Vaccinioideae) including a revision of the genus Comarostaphylis. Ph.D. dissert., University of Wisconsin. Madison, USA. $342 \mathrm{pp}$.

Diggs, G. M. 1995. Comarostaphylis Zuccarini. In: Luteyn, J. L. (ed.). Ericaceae Part II: the superior-ovaried genera. Flora Neotropica, Monograph 66. New York Botanical Garden. New York, USA. pp. 133-193.

González-Elizondo, M., M. S. González-Elizondo y Y. Herrera Arrieta. 1991. Listados florísticos de México. IX. Flora de Durango. Universidad Nacional Autónoma de México. México, D.F., México. 167 pp.

González-Elizondo, M. S. y M. González-Elizondo. 2014. Ericaceae. Flora del Bajío y de regiones adyacentes 183: 1-123.

González-Espinoza, M., J. A. Meave, F. G. Lorea-Hernández, G. Ibarra-Manríquez y A. C. Newton. 2011. The red list of Mexican cloud forest trees. Fauna \& Flora International. Cambridge, UK. 149 pp.

González-Villarreal, L. M. 1990. Las ericáceas de Jalisco, México. Colección Flora de Jalisco. Instituto de Botánica, Universidad de Guadalajara. Guadalajara, México. 140 pp.

IUCN. 2013. IUCN Red list of threatened species. Version 2013.2. <www.iucnredlist.org>. Downloaded on 24 March 2014.

Luteyn, J. L., R. L. Wilbur and L. J. Dorr. 2009. Ericaceae. In: Davidse, G., M. Sousa S., S. Knapp and F. Chiang. (eds.). Flora Mesoamericana 4(1): 411-463.

Martínez-Camilo, R., M. A. Pérez-Farrera y N. Martínez-Melendez. 2012. Listado de plantas endémicas y en riesgo de la Reserva de la Biosfera El Triunfo, Chiapas, México. Bot. Sci. 90(3): 263-285.

Martínez-Gordillo, M., R. Cruz-Durán, J. F. Castrejón-Reyna, S. Valencia-Ávalos, J. JiménezRamírez y C. A. Ruiz-Jiménez. 2004. Flora vascular de la porción guerrerense de la Sierra de Taxco, Guerrero, México. Anales Inst. Biol. Univ. Nac. Autón. México, ser. Bot. 75(2): 105-189.

Rodríguez, A., A. K. Monro, O. Chacón, D. Solano, D. Santamaría, N. Zamora, F. González y M. Correa. 2011. Regional and global conservation assessments for 200 vascular plant species from Costa Rica and Panama. Phytotaxa 21: 1-216.

Osmundson, T. W., R. E. Halling y H. C. den Bakker. 2007. Morphological and molecular evidence supporting an arbutoid mycorrhizal relationship in the Costa Rican paramo. Mycorrhiza 17(3): 217-222.

Stevens, W. D., C. Ulloa-Ulloa, A. Pool y O. M. Montiel. 2001. Flora de Nicaragua. Monogr. Syst. Bot. Missouri Bot. Gard. 85: 1-943.

Zacarías-Eslava, Y. y R. F. del Castillo. 2010. Comunidades vegetales templadas de la Sierra Juárez, Oaxaca: pisos altitudinales y sus posibles implicaciones ante el cambio climático. Bol. Soc. Bot. Méx. 87: 13-28. 